\title{
Structure and dynamics of water at step edges on the calcite $\{10 \overline{1} 4\}$ surface
}

\author{
Marco De La Pierre,$^{1 *}$ Paolo Raiteri, ${ }^{1}$ and Julian D. Gale 1 \\ ${ }^{1}$ Curtin Institute for Computation, The Institute for Geoscience Research (TIGeR), \\ Department of Chemistry, Curtin University, PO Box U1987, Perth, Western Australia 6845, \\ Australia
}

Keywords: calcium carbonate, biomineralization, molecular dynamics simulation, force field calculations, step growth rate, water residence time, water exchange rate

\footnotetext{
* corresponding author: marco.delapierre@curtin.edu.au
} 


\begin{abstract}
The behavior of liquid water around obtuse and acute steps parallel to $<\overline{4} 41>$ on the $\{10 \overline{1} 4\}$ cleavage surface of calcite has been investigated by means of classical molecular dynamics simulations performed with a force-field fitted against thermodynamic properties. Water density maps, radial distribution functions and water average residence times have been investigated. The structure and dynamics of the first two ordered hydration layers, which have been previously observed for the basal surface of calcite, are found to be disrupted by the presence of the steps over a range of five molecular rows either side of the step edge. Calcium sites along the step top edge can coordinate up to three water molecules, as compared with just the single water that can be adsorbed per calcium ion on the flat surface. Water residence times at calcium sites in the vicinity of the step span greater than 2 orders of magnitude, from tenths to several tens of ns, as compared to 2 ns and 0.2 ns for the flat surface and a calcium ion in aqueous solution, respectively. The occurrence of waters with long residence times at the step corners points towards the possible role of step dehydration as a rate-limiting factor in calcite crystal growth. Indeed, the different distributions of slow and fast waters along the obtuse and acute steps appear to correlate with the different rates of growth observed for the two types of steps.
\end{abstract}




\section{Introduction}

Calcite is one of the minerals whose dissolution and growth has been most extensively studied to date. Not only is it important in the context of developing a fundamental understanding of crystallization, but this system is also relevant to processes including biomineralization, ${ }^{1}$ carbon sequestration ${ }^{2}$ and industrial scale inhibition. ${ }^{3}$ The advent of Atomic Force Microscopy (AFM) in the late 1980s made it possible to directly image the surface of calcite growing in aqueous solution. Although it is still a matter of debate whether fully atomistic resolution of individual surface ions can be achieved in water, extended features such as step edges can be clearly identified and their position monitored as a function of time. This makes calcite an important system for the development of theories for the dissolution and growth of minerals based on the step velocities measured during AFM experiments at various levels of super- or under-saturation (e.g. see Ref [ $\left.{ }^{4}\right]$ ).

At ambient conditions calcite is the thermodynamically stable polymorph of calcium carbonate $\left(\mathrm{CaCO}_{3}\right)$ and has a rhombohedral crystal structure with the space group $\mathrm{R} \overline{3} \mathrm{c}$. The predominant cleavage plane, $\{10 \overline{1} 4\}$, displays two different types of monoatomic steps parallel to $\langle\overline{4} 41>$, conventionally named acute and obtuse due to the different inclinations assumed by the carbonate ions in the as cleaved structure. Calcite can be routinely produced in the laboratory by controlled solution chemistry and it naturally displays a high density of screw dislocations on its basal plane, which are a continuous source of steps that can be easily tracked by AFM during growth experiments. This makes calcite the perfect system to develop and test kinetic rate laws to describe the step nucleation and propagation as a function of the solution parameters (supersaturation, $\mathrm{pH}, . .$. ).5,6 The effect of additives and varying ionic strength has also been extensively investigated.7-9

These mechanistic models have been successful in describing the phenomenological behavior observed during the experiments, however they lack the atomistic details of the 
processes occurring at the mineral-water interface. In particular, they do not allow the rationalization in terms of the varying desolvation rates that can be shown by different ions in solution, as recognized by Zhang and Nancollas, ${ }^{10}$ or by non-equivalent surface steps, as postulated by Ruiz-Agudo et al. ${ }^{9}$ to interpret the observed dependence of the anisotropy of obtuse/acute step kinetics upon the $\mathrm{Ca}^{2+} / \mathrm{CO}_{3}{ }^{2-}$ activity ratio in solution. ${ }^{11,12}$ Dehydration of either the ions in solution or the surface sites could, in many cases, represent the ratelimiting process for step nucleation and growth.

Following the observations by Zhang and Nancollas, ${ }^{10}$ Stack and Grantham ${ }^{12}$ proposed a rate model for the growth of calcite where step propagation was described in terms of independent adsorption rates for calcium and carbonate. They concluded that calcite growth is controlled by the ligand-exchange reaction at the solvated ions, while dissolution is limited by the rate at which the "bond" with the surface is broken. Subsequently Wolthers et al. ${ }^{13}$ expanded on this idea and tried to build a kinetic model for the growth of calcite using water exchange rates at surface sites extracted from atomistic computer simulation. In their approach the adsorption rates of the anions were assumed to depend on the desolvation rate of the various surface calcium sites. Although the assumptions behind this kinetic model are reasonable, and seem to be able to reproduce the experimental observations, the reported water exchange rates at the various surface sites appear to be far higher than those that would be expected based on a hypothesis that ligand exchange is rate-limiting for growth. Most of the calcite surface sites were reported to have water mean residence times shorter than 10 ps, with the longest residence time, 42 ps, being observed for the Ca ions on the flat surface. To put these numbers into context, it is important to note that in pure water hydrogen bond exchange happens on a 2-10 ps timescale. ${ }^{14}$ Hence the results of this earlier study would imply that the surface of calcite had little or no effect on the dynamics of water relative to the bulk liquid environment. 
In earlier computational works by by Kerisit and Parker ${ }^{15}$ and Cooke and Elliot ${ }^{16}$ the water residence times at various Ca sites on the calcite surface, as well as at isolated ions, step edges and corners of calcite nanoparticles, were also calculated with reported values between 30 and 300 ps. Again the computed water residence time for some sites appears to be rather short, suggesting that water is able to exchange on timescales only slightly longer than hydrogen bond exchange in bulk water. ${ }^{14}$ One possible cause of these seemingly anomalous results is the polarizable force-field chosen by both research groups. Specifically, Wolthers et al. ${ }^{13}$ used a water model that has been shown to freeze at ambient conditions. ${ }^{17,18}$ While Cooke and Elliot ${ }^{16}$ employed a modified version of the same model that no longer freezes under the same conditions, ${ }^{15}$ this model fails to address the other significant weaknesses of the force-field, such as the equilibrium density of water $(1.27$ $\left.\mathrm{g} / \mathrm{cm}^{3}\right)$, the number of water molecules in the first coordination shell $(\sim 12)$ and the overstructuring of the liquid phase, as shown by the presence of oscillations in the radial distribution function that extend beyond $15 \AA .{ }^{19}$ Although one would have expected that a model that leads to slowing dynamics and over-structuring should result in slower water exchange rates, the unrealistic large number of water molecules in the first coordination shell increases the probability of observing exchange events. Furthermore, some of these events may be faster than for the core 6-8 waters usually believed to be associated with $\mathrm{Ca}^{2+}$ in water and, hence a faster exchange rate is observed.

In several experimental and computational works the first water hydration layer on minerals and metals is sometimes referred to as "ice-like", ${ }^{20-22}$ which suggests a high degree of lateral order and a limited mobility of the molecules. This contrasting description of the hydration layer, relative to the fast exchange reported in the studies highlighted above, indicates that there is still a gap in our understanding of the structure and dynamics of water at solid-liquid interfaces. In this work we use a recently developed force-field ${ }^{19}$ to 
reexamine the structure and dynamics of water at the calcite step edges and kinks, as compared to the flat surface. Importantly, it will be demonstrated that water exchange rates are far slower than suggested by previous studies, thereby supporting the hypothesis that this can be the rate-limiting step for ion addition to growth sites.

\section{Methodology}

The calcite-water interface was modeled through a periodic cell with dimensions of $125 \AA$ x $153 \AA$ x $70 \AA$, containing 11,040 $\mathrm{CaCO}_{3}$ units and 23,232 water molecules (except for simulations of kinks where $24 \mathrm{CaCO}_{3}$ units were removed). The calcite slab and water layer were approximately 30 and $40 \AA$ A thick, respectively, with the plane of the mineral surface being orthogonal to the z-axis. Half of the top calcite layer was removed to produce a 1-D periodic island along the $\mathrm{x}$-axis with an acute and an obtuse step that are approximately 62 Å apart.

Simulations on kinks were performed using the same cells as described above, except that half of the step was removed from both sides of the terrace, effectively leading to a spacing of about $76 \AA$ between opposite kinks on the same side of the island. Because of the crystal structure and symmetry of calcite, 16 unique types of kink can be constructed on the basal plane. Hence, four separate simulation cells were constructed with four different kinks displayed in each case; 2 on the acute and 2 on the obtuse steps.

Classical molecular dynamics simulations for this system were run using the LAMMPS package. ${ }^{23}$ After an initial relaxation of the simulation cell in the $\mathrm{z}$ direction, production runs were performed in the canonical ensemble with a $1 \mathrm{fs}$ timestep. The temperature was kept at $300 \mathrm{~K}$ via a Nosé-Hoover chain of thermostats (length 5) with a relaxation time of 0.1 ps. Based on this set up, we performed a single run of $160 \mathrm{~ns}$, in order to calculate the water residence time at the step edges to within a satisfactory level of statistical uncertainty for those sites with residence times longer than a few nanoseconds. The dynamics of water at 
the kink sites was monitored during a 60 ns run (one simulation per cell). In all simulations atomic trajectories were collected every 1 ps.

In the present work the atomic interactions were described using the force-field reported in Ref. $\left.{ }^{19}\right]$, which has been developed by carefully fitting the thermodynamic properties of the alkaline-earth carbonate minerals. During the fitting of this force-field several important quantities were targeted in order to provide an accurate description of the mineral-water interactions, including the hydration free energy of the alkaline-earth cations and of carbonate, as well as the solubility of the mineral phases. The SPC/Fw water model ${ }^{24}$ has been taken from the literature due to its simplicity and ability to capture some of the key properties of water at ambient conditions, such as the experimental pair distribution function, dielectric constant and hydrogen bond dynamics. ${ }^{24}$ The SPC/Fw model has also proved to be robust and transferable to high temperatures ${ }^{19}$ as well as in supercritical conditions. ${ }^{25}$ In particular, the potential developed by Raiteri et al. ${ }^{19}$ has been shown to reproduce the experimental entropic contribution of the hydration free energy of carbonate and the alkaline-earth ions, which is strongly related to the water structure and dynamics around the ions.

The molecular trajectories from the MD simulations were then analyzed by calculating water 3D density maps, radial distribution functions and water residence times. Each surface calcium site was analyzed independently and the properties of those ions in symmetry equivalent positions were then averaged together in order to improve the statistics.

In principle, the key quantity required to estimate the average residence time of water molecules at the surface calcium sites is the corresponding distribution function $E(t)$, with $E(t) d t$ representing the probability for a water molecule to remain coordinated to a calcium site for a timespan included within $t$ and $t+d t$. However, in order to work with a function 
that contains less noise it is advisable to use the survival function $P(t)$ instead, which is defined as the following integral of $E(t)$ (see e.g. $\left.\left.{ }^{26}\right]\right)$ :

$P(t)=\int_{t}^{\infty} E\left(t^{\prime}\right) d t^{\prime}$

Here, the probability for a water molecule to remain coordinated to a calcium site for $a t$ least a time included within $t$ and $t+d t$ is equal to $1 / \tau P(t) d t$ (with $\tau$ being the average residence time, unknown a priori). Once this survival function $P(t)$ was extracted from the trajectory of the simulation, the average water residence times, $\tau_{i}$, were then calculated by fitting it with a sum of exponentially decaying functions: ${ }^{27}$

$P(t)=\sum_{i=1}^{m} a_{i} \cdot e^{-t / \tau_{i}}$

Depending on the calcium site, we used between one and three exponentials $(m=1-3)$ to capture the water behavior at that site. Radial distribution functions were computed at each calcium site with respect to water oxygen atoms and the position of the first minimum was taken as the cut-off radius to decide whether a water molecule belonged to the first coordination sphere of the site or not. In addition, the integral of the first peak was inspected to infer the number of coordinated waters, and thus of required exponentials; in cases where two water molecules had almost identical residence times the number of exponential functions was reduced by one to remove instabilities in the fitting procedure. As per standard practice, ${ }^{27}$ we applied a threshold period of 1 ps before a water molecule is considered to have left the calcium coordination shell. In this study the water librations were excluded by fitting only the tail of the survival function from 70 ps onwards. The fits were performed between 70 ps and 6 ns where noise in the function was still acceptable. Only in two cases (L1 site 2 (acute) and L1 site 2 (obtuse), see later for nomenclature) this range was found to be inadequate to capture the water dynamics and so the survival function was fitted up to $1 \mathrm{~ns}$ and $100 \mathrm{~ns}$, respectively.

\section{Results and discussion}




\section{Water density maps}

It has long been recognized both experimentally ${ }^{28-30}$ and computationally ${ }^{15,31}$ that water forms two relatively ordered layers on the calcite basal plane. Although the surface unit cell contains two different calcium sites, because of the symmetry of the underlying crystal they have equivalent water solvation structures (and hence share identical properties, such as the water residence time). By looking at the 3D density map of the waters on the calcite flat surface (Figure 1) we can immediately notice that the water molecules in the first layer (yellow iso-density surface) sit with the oxygen on top of the calcium ions while those in the second layer (orange) are localized on top of the carbonates. Considering the position, orientation and relative distances of the water oxygen and hydrogen densities, a hydrogen bond zig-zag pattern can be identified, as described by the blue lines drawn over the top part of Figure 1. According to this picture, the waters of the first layer form hydrogen bonds with the topmost oxygen atoms of the two closest carbonate units. In addition, each second layer water uses the one hydrogen that is aligned on average with the z-axis to establish a hydrogen bond with the same carbonate oxygen atoms.

When a step is created the symmetry is lowered compared to the ideal flat surface (Figure 2), and so it is reasonable to expect different hydration structures (and properties) at the two non-equivalent calcium sites along the step and along the atomic rows in its proximity. Moreover, the surface water structure should progressively converge to the flat surface values as the distance of the calcium sites from the step increases. In Figure 2 the water isodensity surfaces have been made transparent and darker shades been used for increasing density values. This representation provides initial visual, qualitative evidence that from three rows away from the step edge the water arrangement becomes very similar to the one on the pristine flat surface, and that there are sites at the step edge where the water is either more strongly localized (black) or more mobile (white) than on the flat surface. 
Figure 2 also shows the naming convention for the calcite rows that we will use throughout the rest of this paper. For example, the $1^{\text {st }}$ row of atoms in the lower terrace is referred to as "L1", and the $3^{\text {rd }}$ row of atoms in the upper terrace is named "U3".

Figure 3 shows a top view of the density maps at the steps. Notably, because of the smaller angle, and therefore reduced space available, there are less first and second layer waters per unit length along the acute step compared to the obtuse one. In fact, by counting the number of water molecules that coordinate an asymmetric unit of the step edge either on the lower or upper terrace, i.e. considering the sum of waters bound to U1 plus L1 sites, there are 5 (upper) +2 (lower) = 7 first layer waters along the obtuse step, versus 5 (upper) +1 (lower) $=6$ first layer waters along the acute one. Counting second layer waters along the two steps is less trivial, and results in 4 (obtuse) versus 3 (acute, the missing one actually being a poorly localized water above one of the U1 carbonates, namely the one across the figure edges). As regards the first layer waters along row U1 of both steps, note that one out of the two/three coordinated waters at each calcium site is displaced downward, towards the step corner.

Blue bands are again drawn in Figure 3 to help visualize the zig-zag hydrogen bond pattern; here, light blue bands are used to emphasize the fact that this pattern gets altered in the region of the steps. In both the obtuse and acute cases, the patterns on the top and bottom terraces get interrupted at the step, such that some waters will be in "bridging" configurations, hydrogen binding two carbonate units, and other waters will be in "terminal" configurations, binding only one carbonate unit. Another relevant factor is the distance between the U1 and L1 rows in the two type of steps; they are relatively far apart for the obtuse step, such that waters bound to U1 sites do not interact much with ions along row $\mathrm{L} 1$, for example, and the hydrogen bond patterns on the top and bottom terraces are independent from each other. On the other hand, at the acute step the reduced distance 
between the top and corner atomic rows is such that some water molecules can interact with both rows, via electrostatics and/or hydrogen bonds; this is the case for the two waters that coordinate $\mathrm{U} 1$ calcium sites and are displaced downwards.

Finally, we note that along row U1 of the acute step one of the two carbonate units has one oxygen that is displaced downwards away from the surface (circled in light blue in Figure 3) as compared to the flat surface, such that hydrogen bonds with the neighboring water molecules along the same row cannot be formed; as a result, all of the U1 waters along the acute step turn out to be in a "terminal" configuration.

\section{Radial distribution functions}

Radial distribution functions (RDFs) were analyzed for water oxygen atoms around the surface calcium ions. Because the RDF is a radial average around the surface sites, at distances beyond approximately $3 \AA$ the hydration shells of adjacent calcium ions start to overlap, which makes the interpretation of the peaks problematic. However, the position and integral of the first peak (Figure 4) provides information about the average distance and the number, respectively, of water molecules in the first coordination shell. The RDFs reach their first minimum within $3 \AA$ for all sites. However, in the case of site 2 of row L1 of the acute step, the RDF does not get close to zero, unlike in all other cases. Analysis of the water density maps and of the atomic trajectories shows that the water molecule nearest to site 2 of row L1 is actually shared with calcium site 2 at the step top edge (U1), to which it is more strongly bound, such that it constantly moves in and out of the coordination shell of the bottom site along L1. Finally, values of the integral of the first peak of the RDF are larger than one only for calcium sites on the U1 row of both steps; this quantitatively confirms the observation coming from the water density maps that these sites have more than one coordinated water (i.e. either two or three in this case).

\section{Water residence times}


We now turn to the analysis of the average water residence time, $\tau$, as a function of the position of the calcium sites parallel and perpendicular to the calcite surface steps. The survival function, $P(t)$, drops rapidly in the first $20-30 \mathrm{ps}$ (e.g. see the case of the flat surface in Figure 5) at a rate that is comparable to that of bulk water, $\tau \approx 2-10 \mathrm{ps.} \mathrm{This} \mathrm{rapid} \mathrm{water}$ exchange can be assigned to water molecules that never fully enter the calcium coordination sphere, but fluctuate around a distance close to the cut-off radius. Since we are not particularly interested in these fast water exchange events that do not lead to ligand exchange, we fitted only the tail of the survival function above 70 ps. The tail of $P(t)$ for the flat surface (Figure 5, inset) can be perfectly described with a single exponent. In this case the residence time was calculated independently from the survival function of a 960 atom basal surface, as well as for 48 distinct subsets of atoms (each subset containing 12 sites) and found to be $2 \pm 0.2 \mathrm{~ns}$, which is about 10 times longer than $\tau$ for an isolated calcium ion in pure water, $\sim 0.2 \mathrm{~ns}$, based on the current force-field.

Figure 6 shows survival plots on a logarithmic scale for selected calcium sites near the steps. Most of the reported curves are linear, except for three, namely the ones related to site 2 of $\mathrm{U} 1$ (obtuse) and sites 1 and 2 of U1 (acute); these are among the sites that feature either two or three coordinated water molecules (Figure 4 and Figure S2 in the Supplementary Information). Site 1 of L1 (obtuse) and site 1 of L1 (acute) show nearly flat curves in the interval shown, which correspond to residence times as long as $60+$ and $20 \mathrm{~ns}$, respectively (see also Figure S3 in the Supplementary Information). However, with these values being nearly of the same order of magnitude as the total time of the simulation, 160 ns, statistics on the exchange events is poor and a relatively large tail of the residence time distribution function could not even be sampled; these issues result in a larger uncertainty for the survival function and an underestimation of the residence time, respectively. Conversely, site 2 of L1 (acute) shows a very steep curve, suggesting a very short value for $\tau$, 
around $0.15 \mathrm{~ns}$; the water molecule closest to this calcium site is actually the one that upon analysis of the RDF was found to be shared with the site 2 of $\mathrm{U} 1$, to which it is more strongly bound. Such a small value reflects the peculiar dynamics of this water that frequently enters and leaves the coordination sphere of the L1 site while remaining coordinated to the U1 site.

By looking at the full set of residence time values as a function of the distance from the step edge (Table 1 and Figure S1 in the Supplementary Information) we can say the disturbance to the water structure and dynamics caused by the step decays away within 1$1.5 \mathrm{~nm}$ from the edge (i.e. 3 to 5 molecular rows) for both the obtuse and acute steps, with the obtuse step recovering the flat surface behavior earlier than the acute one. This observation matches the qualitative inspection of the 3D water density maps discussed above. In the case of the lower terrace, for up to four rows from the acute step the water

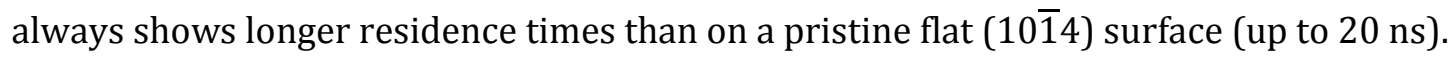
This may have important consequences for the imaging of the steps using AFM, and will be the scope of future work.

In order to better visualize the distribution of the residence time values around the steps, we have taken a representative snapshot of the first water layer on the $(10 \overline{1} 4)$ stepped surfaces and colored the water molecules according to their residence times (Figure 7). In general, the water molecules with the shortest residence times (colored in red, $\tau<0.9 \mathrm{~ns}$ ) are always located at the step top edge while those with the longest residence times (violet, $\tau>10 \mathrm{~ns}$ ) are always at the bottom of the steps. Considering the two atomic rows that make up each of the two steps, along the obtuse one (Figure 7, left panels) there is a clear alternation of "fast" (red/orange) and "slow" (violet/blue) water molecules at calcium sites along both rows $\mathrm{U} 1$ and L1. In contrast, along the acute step (Figure 7, right panels) the top edge (U1) is always surrounded by fast (red) water molecules, whereas at the bottom of the 
step (L1) there are predominantly slow (violet/blue/cyan) water molecules. More details of the atomic structure of first layer waters coordinated to U1 and L1 calcium sites are shown in Figure S4 in the Supplementary Information.

The above observations regarding the water residence time can largely be rationalized based on the analysis of the water density maps that was presented above (see Figure 3). Specifically, long-life sites seem likely to correspond to those water molecules that form a larger number of hydrogen bonds with the surface carbonates, and that exhibit strong interactions with more than one calcium site. In fact, the alternation of fast and slow waters along the obtuse step relates to the occurrence of waters that act as either "terminators" or "bridges" in the hydrogen bond pattern of both U1 and L1 rows. On the other hand, fast waters along row $\mathrm{U} 1$ of the acute step are associated with the condition of "terminators" that is common to all of them; slow waters along row L1 relate to either a "bridging" configuration (for the only water which is top-bound to a L1 calcium site) or to the occurrence of strong electrostatic and hydrogen bond interactions at the same time with sites along both the U1 and L1 atomic rows (for the waters that are side-bound to U1 sites). Note that in this work we have not considered any of the effects due to changes in $\mathrm{pH}$ and ionic strength. Recent work ${ }^{32}$ suggests that carbonate is stable over bicarbonate at the calcite-water interface across the whole $\mathrm{pH}$ range relevant for calcite precipitation, so the neglect of $\mathrm{pH}$ in the present work does not hinder comparison with the experiments. On the other hand, presence of dissolved salts such as alkali halides has been proposed ${ }^{9}$ to stabilize the hydration shell of ions in solutions, and eventually of ions at mineral surfaces, thus slowing the water dynamics; this point will be the subject of future work.

\section{Water dynamics at the kink sites}

Structures for the 16 types of kinks considered for this study are reported in Figures S5 and S6 in the Supplementary Information. If we compare the four simulation cells 
containing the kinks with the one used to study the water dynamics at the steps, it is evident that each symmetry independent kink site appears only once in our model system, as opposed to 12 times in the case of the calcium sites at the steps, and the simulations were only 60 ns long. Overall, this implies that our statistics for the water dynamics are about 20 times smaller for the kink sites than for the sites at the step edges. In addition, more than half of the considered kinks do not preserve a stable structure throughout the simulations. In most cases the ion at the kink extremity, either calcium or carbonate is mobile and it prefers to increase its water coordination number by leaving its crystallographic position and adopting a more open position above the surface at the step edge (e.g. see Figure S7 in the Supplementary Information). Although no dissolution event was observed during the duration of the simulation, having a variable geometry for the kink makes it very difficult to accurately determine the water residence time at the kink sites.

Based on the above observations, we prefer to make some general and qualitative comments on the water behavior rather than to try to extract statistically meaningful water residence times at the kink sites. Firstly, calcium sites at the kink can exhibit a water coordination number of up to 4 in the case of "stable" kinks that conform to the calcite structure, and up to 6 in the case of kinks where the ion adopts a non-crystallographic position above the lower terrace. Secondly, and most importantly, estimated residence times fall in the same range of order of magnitude as observed in the case of step edges, i.e. from tenths to tens of nanoseconds. This suggests that water dynamics at kinks can be at least as slow as that observed at steps.

\section{Conclusions}

The first important conclusion from this study is that water dynamics at the calcite surface can be slow in contrast to previous reports. Indeed, they can actually be more than 2 orders of magnitude slower than the dynamics around free ions in solution, i.e. up to tens of 
ns as compared to hundreds of ps. This implies that in order to estimate the typical residence times with accuracy, relatively long simulations need to be run. Even though it is far more significant to focus on the slow water molecules, i.e. the ones that are bound at the calcium sites, since they are the ones that can affect surface processes the most, it is actually easy to confuse them with the fast, non-bound molecules that rapidly enter and exit the coordination shell. This might be the reason why some previous investigations ${ }^{13,15,22}$ reported very small $\tau$ values, comparable to those for the dynamics of pure water, i.e. $\sim 5-10$ ps. ${ }^{14}$

The surface region within 1-1.5 $\mathrm{nm}$ from a step is where the water structure and dynamics are found to be significantly perturbed with respect to the clean surface. The perturbation of the water structure at these relatively large distances from the step could be one of the factors responsible for the peculiar contrast features that are often observed in high-resolution AFM images of the step edges, and that consist of a few nm wide strip of intermediate height between the top and bottom terraces. 33

Calcium sites at the step top edge allow for a higher water coordination, up to 3 water molecules, and feature the shortest residence times, down to 140 ps. Sites at the step corner show the largest residence times, up to 20 (acute) and 60+ (obtuse) ns, that is 1 order of magnitude larger than the ideal surface ( $2 \mathrm{~ns}$ ) and 2 orders of magnitude larger than ions in solution (200-300 ps); similar large values have been estimated for the kinks, too. Such occurrence of stable, long lived waters at the step could have important consequences on the adsorption of ions and organic molecules, from both thermodynamic and kinetic points of view, as already pointed out by some of us in the case of barite. ${ }^{34,35}$ In particular, dehydration of surface sites is here confirmed to be one of the most relevant potential ratelimiting steps for calcite crystal growth, as already suggested in recent experimental studies with AFM. ${ }^{9}$ Rate constants corresponding to exchange of the waters with the longest 
residence times are $2 \cdot 10^{7}$ and $5 \cdot 10^{7} \mathrm{~s}^{-1}$ at the obtuse and acute steps, respectively. These values therefore would represent an upper bound to propagation of the step edges, since water would have to exchange for any incoming ions. Using data from AFM, Stack and Grantham ${ }^{12}$ have determined that the combined rate constants for step edge nucleation and

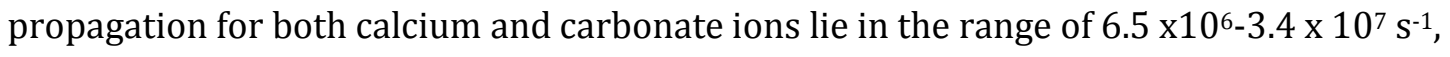
which are consistent with the rates of water exchange found in this study for the slowest sites at the bottom of the step edge.

It is also worth noting that in this study acute and obtuse steps show differences in many respects, including density of water molecules, range of residence time values, distribution of slow and fast water molecules. In particular, this latter microscopic aspect is tackled here for the first time, and could indeed be related to the different growth kinetics affecting the two types of step that have been observed in the experiments. ${ }^{11,12}$ These prior works showed that the $\mathrm{Ca}^{2+} / \mathrm{CO}_{3}{ }^{2-}$ activity ratio affects in different ways the obtuse and acute step spread velocity, such that the kinetics are faster when $\mathrm{Ca}^{2+}$ and $\mathrm{CO}_{3}^{2-}$, respectively, are the dominant species in solution. In addition, they demonstrated that kinetics are, in general, faster at the obtuse rather than at the acute steps; this contrasts with our observation that the slowest exchanging waters are found at the obtuse steps, and suggests that other factors might contribute to the apparent rate-limiting behavior at the acute steps, beside surface dehydration. The occurrence of an alternating sequence of slow and fast waters (obtuse step) versus a more uniform sequence of slow waters (acute) is indeed likely to lead to different kinetics of ion adsorption, featuring different rate-limiting steps. In order to appropriately account for the role of this pattern of slow and fast waters, all the relevant intermediate steps in ion adsorption need to be modeled and investigated. Therefore, further simulations involving the presence of carbonate and calcium ions, as well as ion pairs, in solution are required to shed more light on this important aspect. 


\section{Acknowledgements}

This research was supported by computational resources provided by the Pawsey Supercomputing Centre (funded from the Australian Government and the Government of Western Australia), the Australian National Computational Infrastructure (funded by the Australian Government) and Victorian Life Sciences Computation Initiative (funded by the Government of Victoria). PR and JDG would like to acknowledge the Australian Research Council for funding (FT130100463 and DP160100677).

\section{References}

(1) Meldrum, F. Int. Mater. Rev. 2003, 48 (3), 187-224.

(2) Matter, J. M.; Stute, M.; Snaebjornsdottir, S. O.; Oelkers, E. H.; Gislason, S. R.; Aradottir, E. S.; Sigfusson, B.; Gunnarsson, I.; Sigurdardottir, H.; Gunnlaugsson, E.; Axelsson, G.; Alfredsson, H. A.; Wolff-Boenisch, D.; Mesfin, K.; Taya, D. F. D. L. R.; Hall, J.; Dideriksen, K.; Broecker, W. S. Science 2016, 352 (6291), 1312-1314.

(3) Athanasakou, G. D.; Arvaniti, E. C.; C, A. E.; Koutsoukos, P. G.; Paraskeva, C. A. In Mineral Scales in Biological and Insdustrial Systems; Amjad, Z., Ed.; 2014; pp 3-14.

(4) De Yoreo, J. J.; Vekilov, P. G. Reviews in Mineralogy and Geochemistry 2003, 54, 57 93.

(5) Teng, H. H.; Dove, P. M.; de Yoreo, J. J. Geochim. Cosmochim. Ac. 2000, 64 (13), 22552266.

(6) Ruiz-Agudo, E.; Putnis, C. V.; Rodriguez-Navarro, C.; Putnis, A. Geochim. Cosmochim. Ac. 2011, 75 (1), 284-296.

(7) Elhadj, S.; de Yoreo, J. J.; Hoyer, J.; Dove, P. M. P. Natl. Acad. Sci. USA 2006, 103 (51), 19237-19242.

(8) Ruiz-Agudo, E.; Kowacz, M.; Putnis, C. V.; Putnis, A. Geochim. Cosmochim. Ac. 2010, 74 (4), 1256-1267.

(9) Ruiz-Agudo, E.; Putnis, C. V.; Wang, L.; Putnis, A. Geochim. Cosmochim. Ac. 2011, 75 (13), 3803-3814.

(10) Zhang, J.; Nancollas, G. H. J. Colloid Interf. Sci. 1998, 200, 131-145.

(11) Larsen, K.; Bechgaard, K.; Stipp, S. L. S. Geochim. Cosmochim. Ac. 2010, 74 (2), 558567.

(12) Stack, A. G.; Grantham, M. C. Cryst. Growth Des. 2010, 10 (3), 1409-1413.

(13) Wolthers, M.; di Tommaso, D.; Du, Z.; de Leeuw, N. H. CrystEngComm 2013, 15 (27), 5506-5514.

(14) Ohtaki, H.; Radnai, T. Chem. Rev. 1993, 93 (3), 1157-1204.

(15) Kerisit, S.; Parker, S. C. J. Am. Chem. Soc. 2004, 126 (32), 10152-10161. 
(16) Cooke, D. J.; Elliott, J. A. J. Chem. Phys. 2007, 127 (10), 104706.

(17) Van Maaren, P. J.; Van Der Spoel, D. J. Phys. Chem. B 2001, 105 (13), 2618-2626.

(18) Raiteri, P.; Demichelis, R.; Gale, J. D. Method. Enzymol. 2013, 532, 3-23.

(19) Raiteri, P.; Demichelis, R.; Gale, J. D. J. Phys. Chem. C 2015, 119 (43), 24447-24458.

(20) Toney, M. F.; Howard, J. N.; Richer, J.; Brges, G. L.; Gordon, J. G.; Melroy, O. R.; Wiesler, D. G.; yee, D.; Sorensen, L. B. Nature 1994, 368 (6470), 444-446.

(21) Reedijk, M. F.; Arsic, J.; Hollander, F. F. A.; de Vries, S. A.; Vlieg, E. Phys. Rev. Lett. 2003, 90 (6), 066103-066104.

(22) Kerisit, S.; Cooke, D. J.; Spagnoli, D.; Parker, S. C. J. Mater. Chem. 2005, 15 (14), 1454-1462.

(23) Plimpton, S. J. Comput. Phys. 1995, 117 (1), 1-19.

(24) Wu, Y.; Tepper, H.; Voth, G. A. J. Chem. Phys. 2006, 124 (2), 024503.

(25) Lu, C.-Y.; Perez, D.; Hickmott, D. D.; Voter, A. F. J. Phys. Chem. C 2012, 116 (49), 25934-25942.

(26) Brunne, R. M.; Liepinsh, E.; Otting, G.; Wuthrich, K.; van Gunsteren, W. F. J. Mol. Biol. 1993, 231 (4), 1040-1048.

(27) Impey, R. W.; Madden, P. A.; McDonald, I. R. J. Phys. Chem. 1983, 87 (25), 50715083.

(28) Geissbuhler, P.; Fenter, P.; DiMasi, E.; Srajer, G.; Sorensen, L.; Sturchio, N. C. Surf. Sci. 2004, 573 (2), 191-203.

(29) Watkins, M.; Reischl, B. J. Chem. Phys. 2013, 138 (15), 154703.

(30) Marutschke, C.; Walters, D.; Hermes, I.; Bechstein, R.; Kühnle, A. Nanotechnology 2014, 25 (33), 335703.

(31) Fenter, P.; Kerisit, S.; Raiteri, P.; Gale, J. D. J. Phys. Chem. C 2013, 117 (10), 50285042.

(32) Andersson, M. P.; Rodriguez-Blanco, J. D.; Stipp, S. L. S. Geochim. Cosmochim. Ac. 2016, 176 (C), 198-205.

(33) Fukuma, T.; Reischl, B.; Kobayashi, N.; Spijker, P.; Canova, F. F.; Miyazawa, K.; Foster, A. S. Phys. Rev. B 2015, 92 (15), 155412-155417.

(34) Piana, S.; Jones, F.; Gale, J. D. J. Am. Chem. Soc. 2006, 128 (41), 13568-13574.

(35) Stack, A. G.; Raiteri, P.; Gale, J. D. J. Am. Chem. Soc. 2012, 134 (1), 11-14. 
Tables

Table 1. Water residence times (ns) at the calcium adsorption sites of obtuse and acute steps of the $(10 \overline{1} 4)$ calcite surface.

\begin{tabular}{|c|c|c|c|c|}
\hline \multirow{2}{*}{ Row } & \multicolumn{2}{|l|}{ Obtuse } & \multicolumn{2}{|l|}{ Acute } \\
\hline & Site 1 & Site 2 & Site 1 & Site 2 \\
\hline \multicolumn{5}{|l|}{ Upper } \\
\hline \multirow[t]{3}{*}{1} & 6.3 & 1.2 & 4.0 & 3.0 \\
\hline & " & 0.50 & 0.44 & 0.60 \\
\hline & & " & 0.14 & \\
\hline 2 & 2.8 & 1.2 & 4.6 & 0.93 \\
\hline 3 & 3.5 & 2.0 & 1.4 & 1.4 \\
\hline 4 & 2.2 & 1.6 & 2.4 & 2.3 \\
\hline 5 & 2.1 & 2.1 & 2.1 & 1.8 \\
\hline 6 & 2.3 & 2.0 & 2.1 & 2.0 \\
\hline 7 & 2.4 & 2.0 & 2.1 & 1.8 \\
\hline 8 & 2.0 & 2.0 & 2.0 & 2.0 \\
\hline 9 & 2.1 & 2.0 & 1.9 & 1.9 \\
\hline 10 & 1.9 & 1.7 & 2.1 & 2.0 \\
\hline \multicolumn{5}{|c|}{ Lower } \\
\hline 1 & $60+$ & 1.3 & 20 & $(0.15)^{*}$ \\
\hline 2 & 2.1 & 2.1 & 5.2 & 3.7 \\
\hline 3 & 2.5 & 2.1 & 3.2 & 2.3 \\
\hline 4 & 2.2 & 2.0 & 2.6 & 2.4 \\
\hline
\end{tabular}




\begin{tabular}{|l|l|l|l|l|}
\hline 5 & 2.1 & 1.9 & 1.9 & 1.8 \\
\hline 6 & 2.2 & 1.9 & 2.2 & 2.1 \\
\hline 7 & 2.0 & 2.0 & 2.2 & 1.9 \\
\hline 8 & 2.0 & 2.0 & 2.2 & 2.2 \\
\hline 9 & 2.1 & 1.9 & 2.0 & 1.9 \\
\hline 10 & 1.9 & 1.9 & 2.2 & 1.8 \\
\hline
\end{tabular}

The first ten rows of the upper (U) and of the lower (L) terraces were considered, where increasing number implies a greater distance from the step. The two symmetry independent sites were treated separately. For each row, site 1 has been chosen as the one featuring the longest residence time. The computed residence time for the flat surface is 2.0 ns. Sites along row U1 host more than one water molecule, and therefore multiple values are given over 3 successive lines of the table. ${ }^{*}$ The water closest to site 2 of row L1 coincides with the first of the two waters coordinated to site 2 of row $\mathrm{U} 1$. 


\section{Figures}
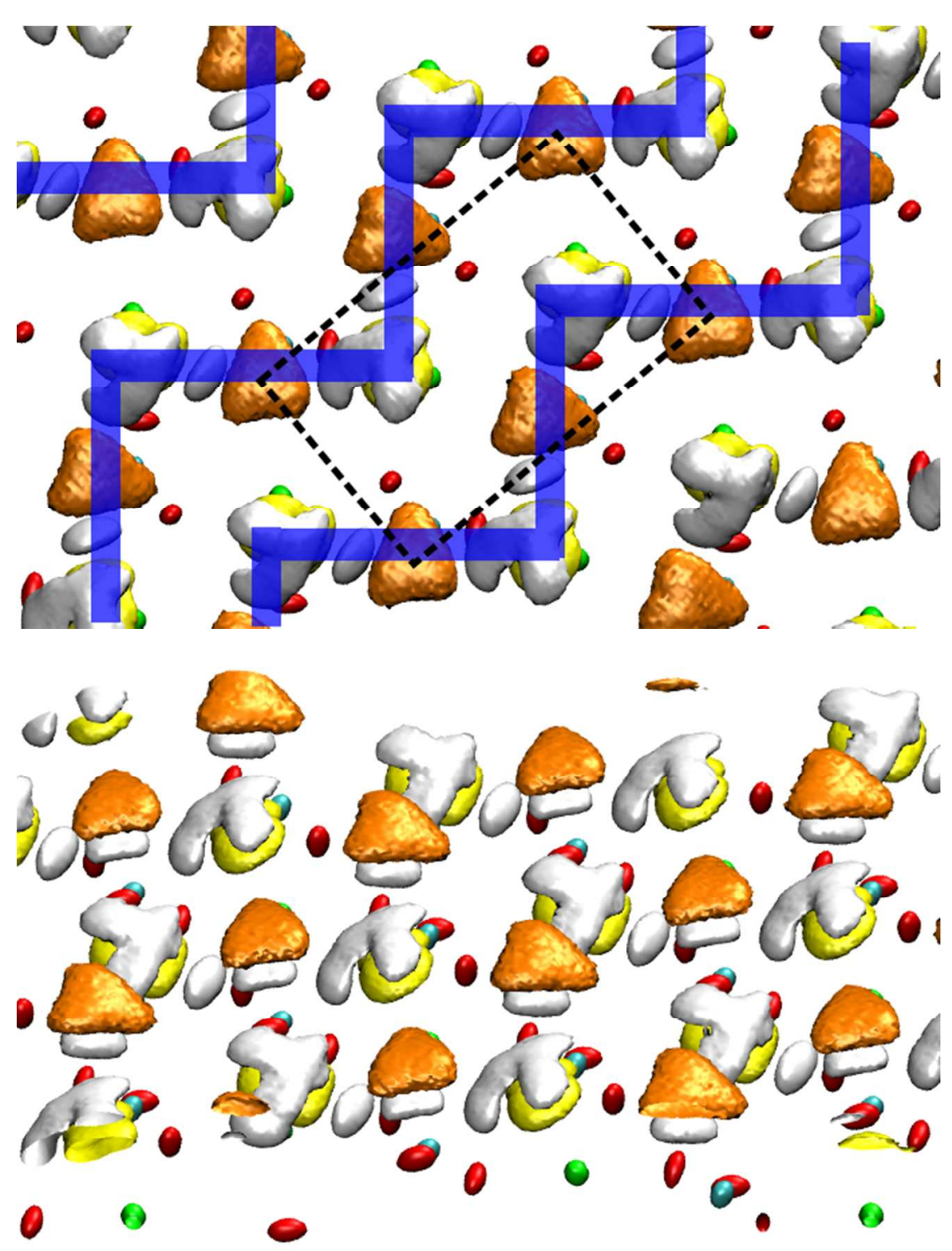

Figure 1: Top (upper) and tilted (lower) views of the 3D density map of the calcite basal surface and adjacent water layers. The tilting is by $45^{\circ}$ around the [ $\left.\overline{4} 41\right]$ crystallographic direction, i.e. the direction parallel to the steps under study. The iso-density surfaces are colored based on the atom types; green is used for $\mathrm{Ca}$, red for the oxygen atoms of carbonate, and yellow for the oxygen atoms of the waters in the first hydration layer. Cyan is used for the carbon atoms, though they are almost completely obscured by the oxygen of the water molecules in the second hydration layer, shown in orange. Finally, white surfaces 
refer to hydrogen atoms of the first two water hydration layers. Blue lines are drawn as a guide to the eye along the hydrogen bond patterns involving the calcite surface and the first water layer. The unit cell of the calcite surface is shown with a dashed line. 

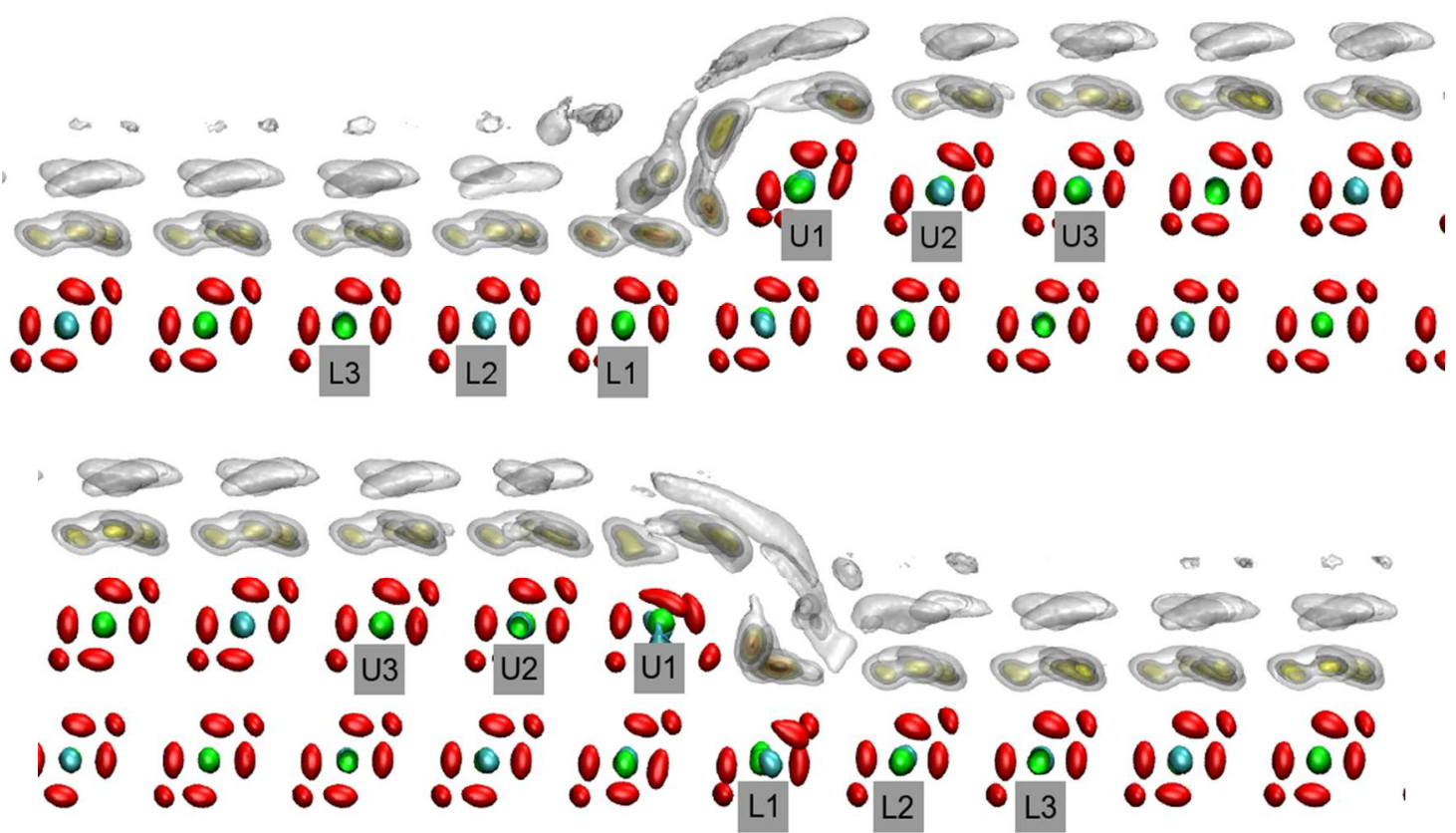

Figure 2. Side view of the 3D atomic density maps at the obtuse (top) and acute (bottom) calcite steps parallel to the $[\overline{4} 41]$ crystallographic direction on a $(10 \overline{1} 4)$ calcite surface. The iso-density surfaces have been colored based on the atoms' type, with green for Ca, red for the carbonate oxygen atoms and cyan for carbon. Because of the difference in height between the terraces it is difficult to discriminate between the first and second water layer on the basis of position of the atoms, so instead the water-oxygen iso-density surfaces, shown as transparent and darker colours, have been used for higher density regions (white, grey, yellow, orange and black in order of increasing density: 2, 10, 20, 40 and 67 atoms $/ \AA^{3}$ ). Superimposed labels give the adopted notation for atomic rows in the proximity of the steps. 


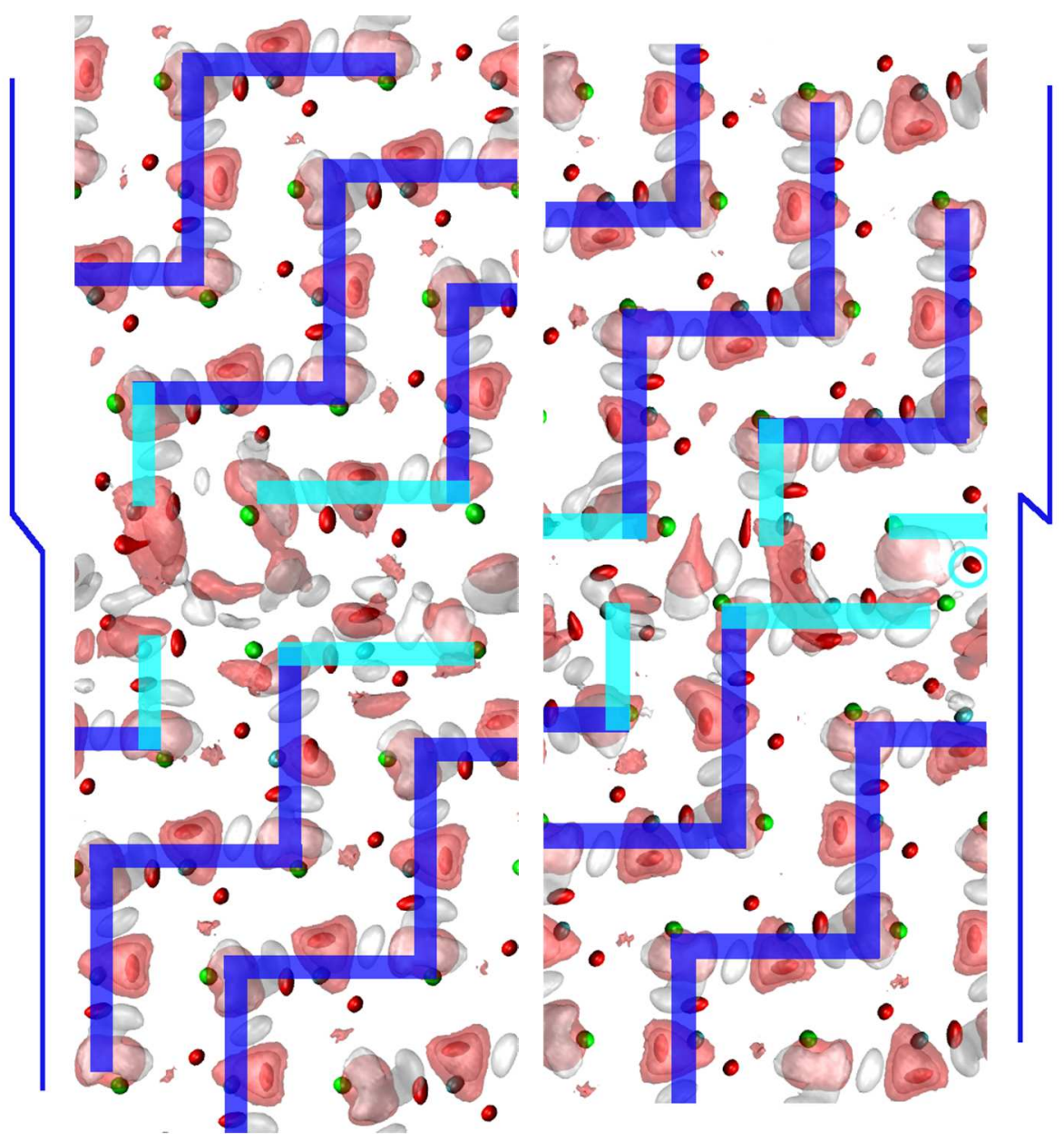

Figure 3. Top view of the 3D atomic density maps at the obtuse (left) and acute (right) calcite steps. The iso-density surfaces have been colored based on the atoms' type, with green for Ca, red for the oxygen atoms of carbonate and cyan for carbon. Iso-density surfaces corresponding to both oxygen and water atoms of the first two water hydration layers are shown here in transparent red and white colors, respectively. Thick dark blue lines are drawn as a guide to the eye in order to highlight the hydrogen bond patterns involving the calcite surface and the first water hydration layer; light blue is used to emphasize the disruption of these patterns, relative to the terraces, in the region of the steps. The light blue circle highlights an oxygen in a carbonate unit along the acute step that undergoes a significant lowering orthogonal to the surface, as compared to flat calcite. The 
two thin blue vertical lines indicate the position of the step edges relative to the iso-density surfaces. 


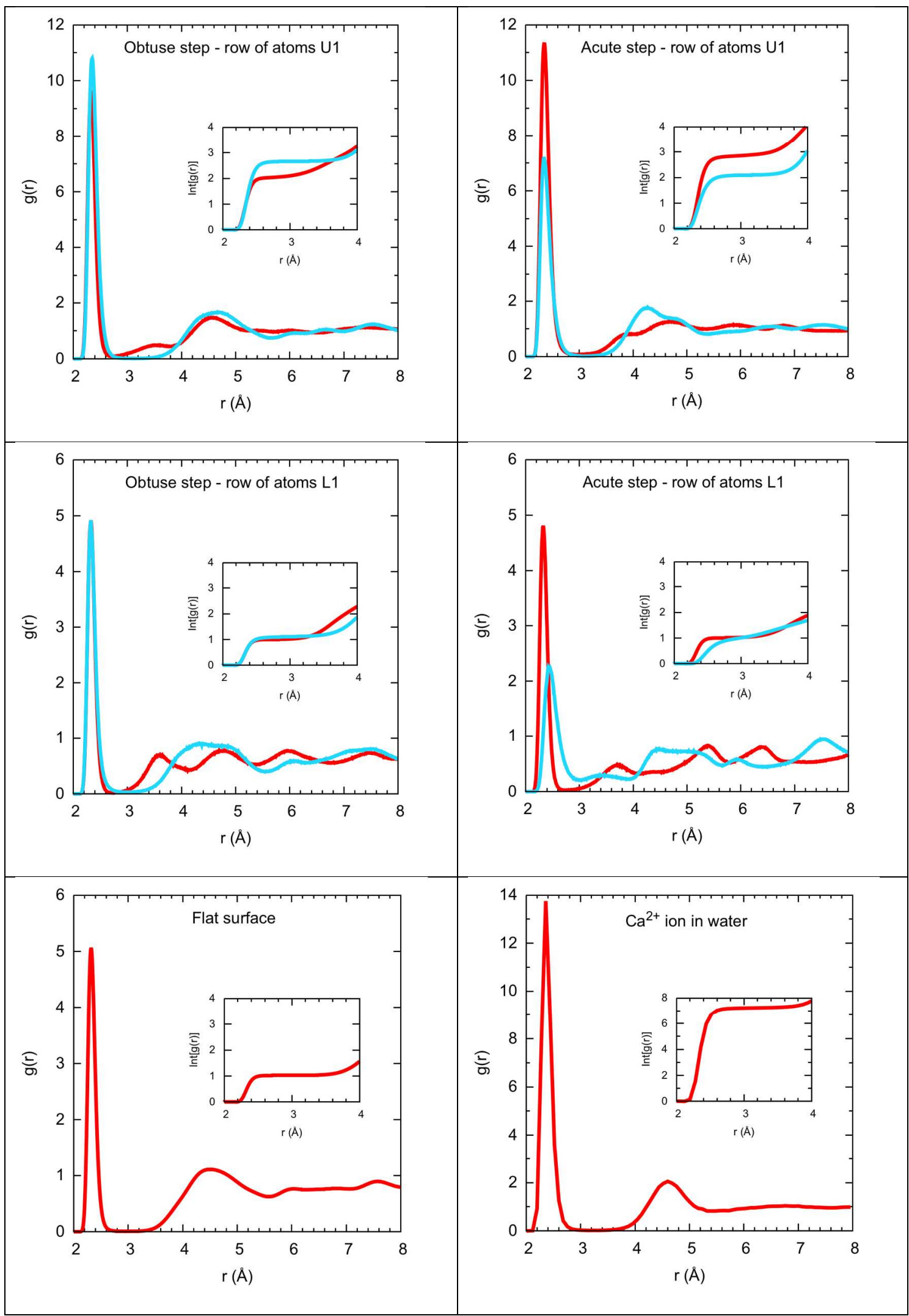


Figure 4. Radial distribution functions of the oxygen atoms of water around a set of surface calcium sites in proximity to the obtuse and acute step edges, as compared to a calcium site on a flat surface, and to a calcium ion in aqueous solution. Insets show the integrals of the distribution function at short distances, which plateau to the coordination numbers at the corresponding sites. Red and blue curves refer to sites 1 and 2, respectively, while U1 indicates a site in row 1 of the upper terrace and L1 a site in the corresponding row on the lower terrace. 


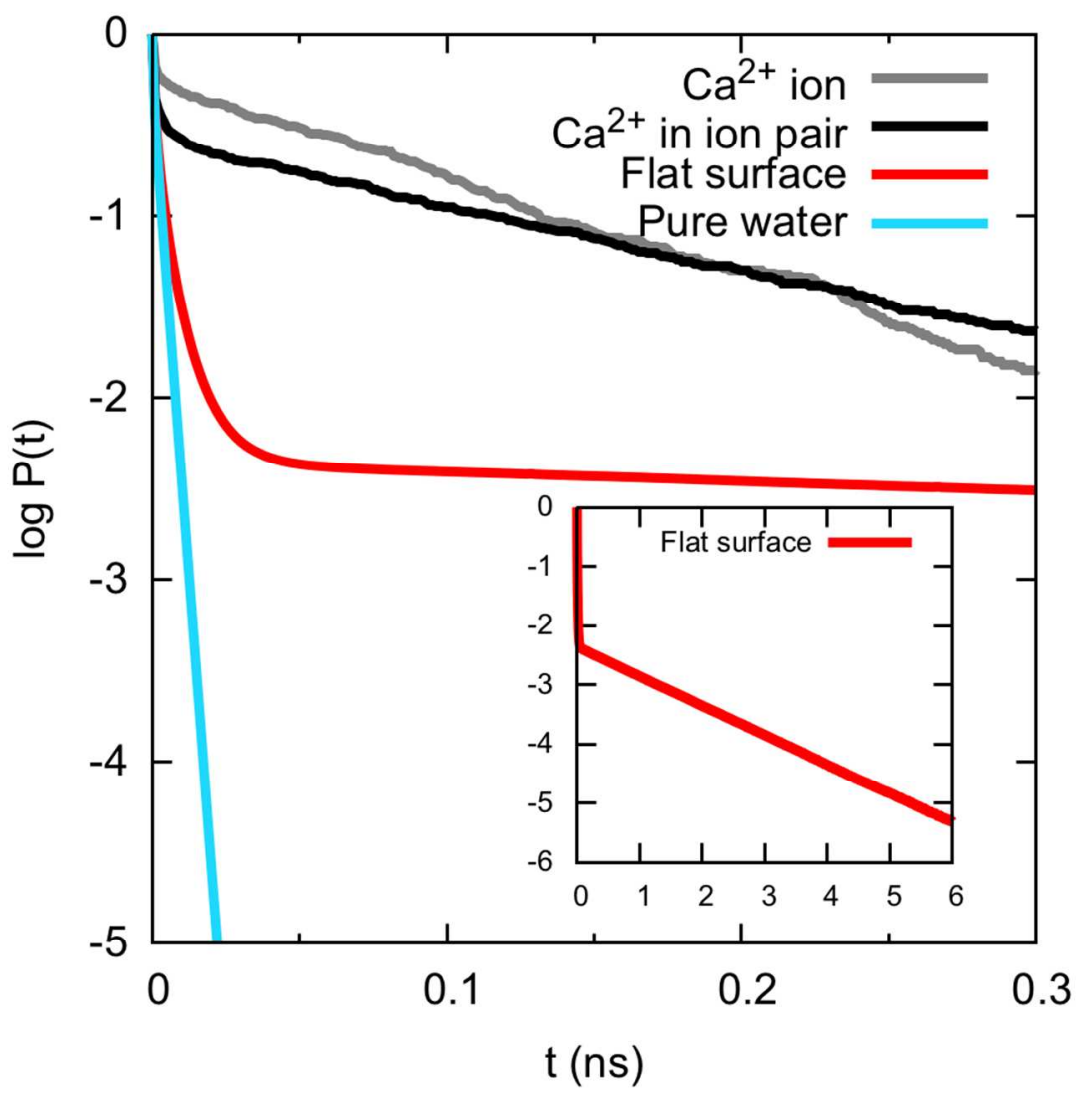

Figure 5. Survival function (logarithmic scale) for water molecules around the calcium sites of a flat calcite (1014) surface, as compared to a calcium ion in aqueous solution, a calcium cation in an ion pair with carbonate in solution, and pure water. The main panel shows data for the initial 300 ps, while the inset shows the function for the flat surface over a longer 6 ns time period. 


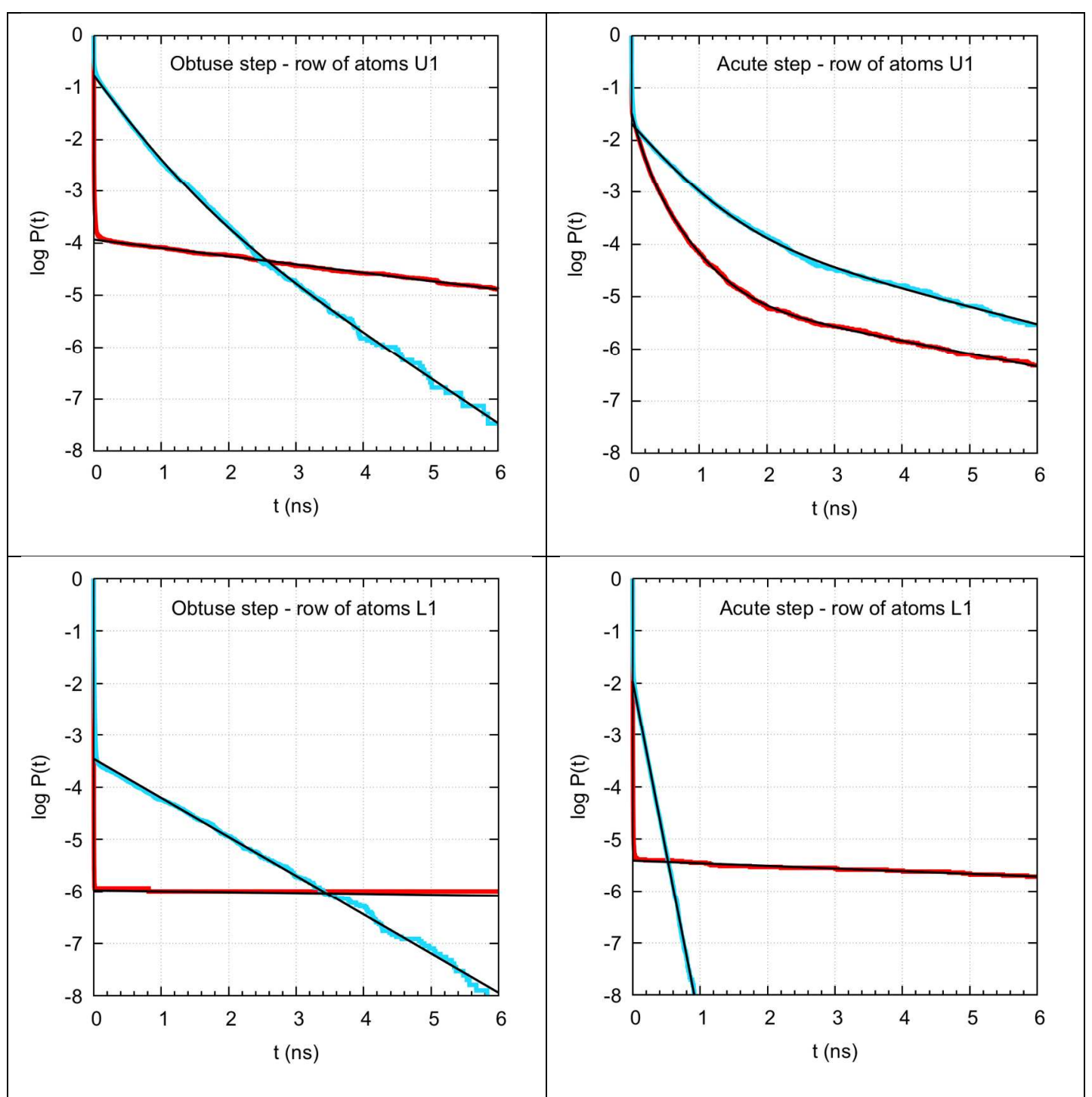

Figure 6. Survival function (logarithmic scale) for water molecules for a set of calcium sites in proximity to the obtuse and acute step edges. Red and blue curves refer to sites 1 and 2, respectively. Best-fit curves are shown in black. 


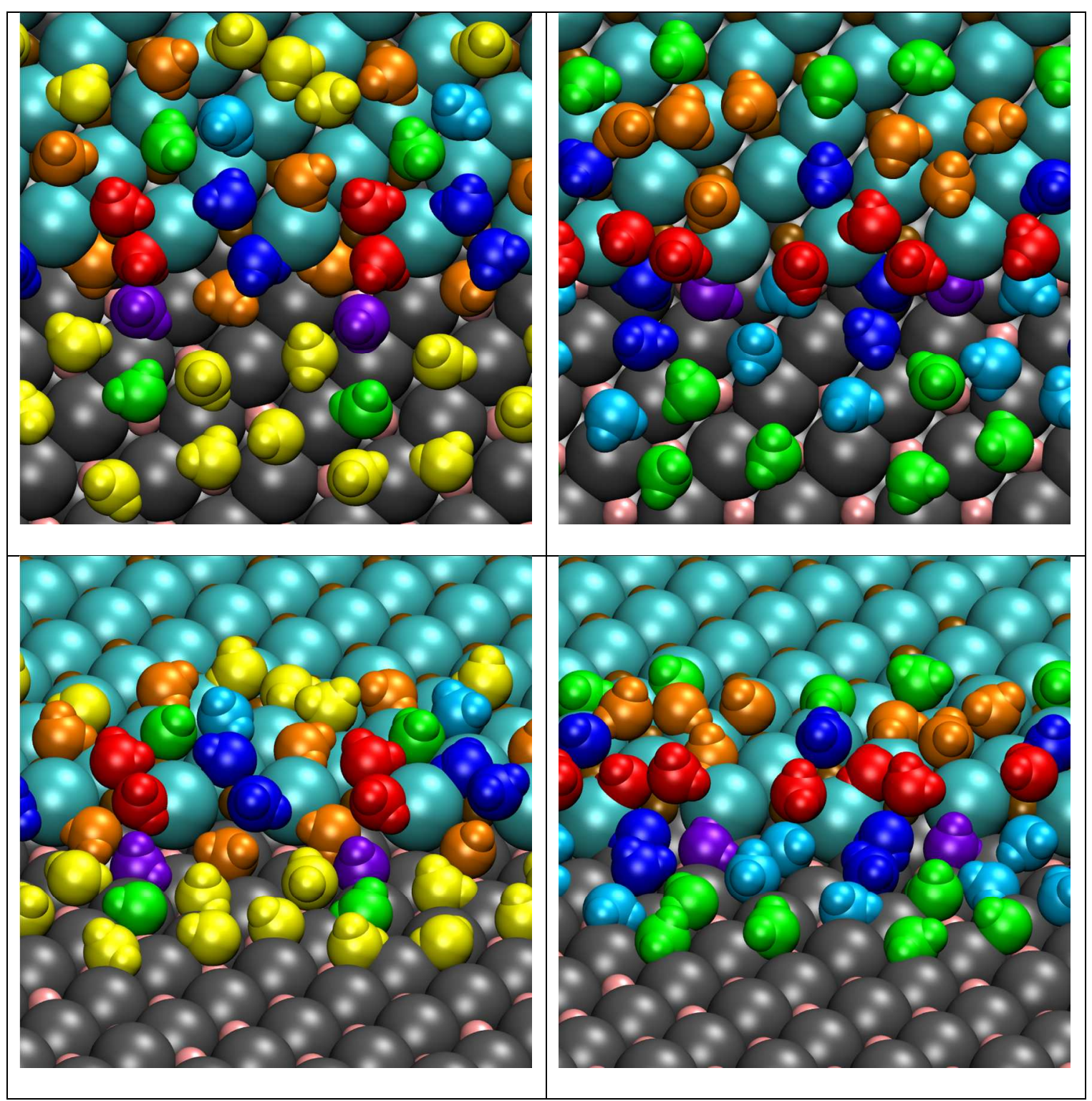

Figure 7. Top down (upper) and tilted (lower) views of the obtuse (left) and acute (right)

steps. In the upper terrace, calcium ions and carbonate units are represented as ochre and

cyan spheres, respectively; in the lower terrace, they are in pink and grey, respectively.

Water molecules coordinated to the calcium sites have been shown up to those bound to the fourth row from the step. Molecules are colored according to their residence time using the following criteria; red < 0.9 ns; orange 0.9-1.8 ns; yellow 1.8-2.3 ns; green 2.3-3 ns; cyan 3-4 ns; blue 4-7 ns; violet > 10 ns.

\section{For Table of Contents Use Only}


The distribution of water exchange rates along obtuse and acute steps of a (10 $\overline{1} 4)$ calcite surface is obtained through accurate classical molecular dynamics simulations and discussed in relation with the kinetics of crystal growth.
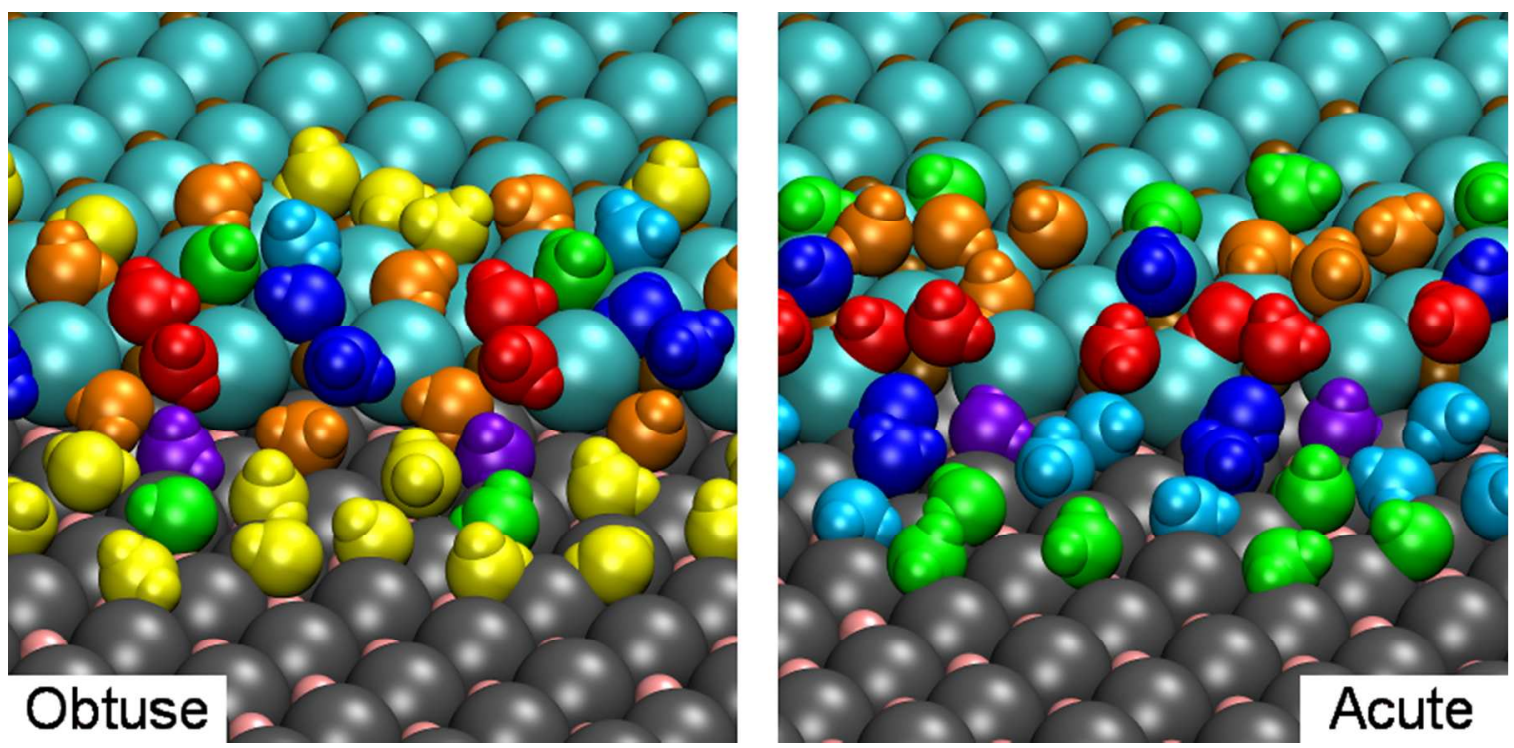

Acute 